\title{
Graphical deployment strategies in radar sensor networks (RSN) for target detection
}

\author{
Ling Yang ${ }^{*}$, Jing Liang ${ }^{*}$ and Weiwei Liu
}

\begin{abstract}
Nodes deployment is a major challenge to a successful implementation of radar sensor network (RSN). The goal of the deployment is to ensure that the target can achieve expected detection performance with high-energy efficiency. In this article, two deployment strategies, named Hexagonal Deployment Strategy (HDS) and Diamond Deployment Strategy (DDS), are proposed to solve this problem. Each Radar Sensor (RS), separately, obtains probability of target detection depending on the position of RS according to the deployment strategies. To appraise these two deployment strategies, two decision fusion rules are derived over pass-loss fading channel in multi-hop RSN. We combine these two decision fusion rules with unfixed local detection performance. Simulations results show that given a finite number of RSs, our proposed strategies are far superior to Random Deployment Strategy in terms of detection probability and energy consumption to satisfy detection and false alarm requirements. The DDS achieves higher probability of detection and consumption fewer energy than HDS, no matter in decision fusion rules with Binary Transmission (BT) or without Binary Transmission (NBT). The BT fusion rules performs better than NBT and the number of RS needed for expected detection performance is not the more the better.
\end{abstract}

Keywords: Graphical deployment strategies, Decision fusion rules, Radar sensor networks, Target detection, Energy efficiency

\section{Introduction}

Radar Sensor Networks (RSN) have attracted growing interest in various applications. They can monitor a large area and observe targets from many different angles. These networks will be included in the tactical combat systems that are deployed on airborne, surface, and subsurface unmanned vehicles in order to protect critical infrastructure from terrorist activities [1-3]. In a resourceconstrained RSN, a deployment strategy is used to ensure that targets in the sensor field can be detected properly and the energy consumed should be as little as possible.

For target detection problem in RSN, Ly and Liang [4] exhibited a diversity scheme to improve detection performance of RSN in the presence of strong interference, especially clutters and noise, and then proposed a maximum likelihood multi-target detection algorithm to estimate the possible number of targets in a surveillance area in RSNs [5]. Deng [6] introduced a distributed

*Correspondence: yangling891@126.com; liangjing@uestc.edu.cn School of Electrical Engineering, University of Electronic Science and Technology of China, Chengdu, China
RSNs systems for effective target detection and the detection performance of this system improves dramatically with the increase of the number of radar sensors (RSs) in the system. Shu and Liang [7] studied the decision fusion rules of multiple fluctuating targets in multi-radar sensor networks under multi-hop transmission. They all discussed the detection performance and considered the information integration in RSN. However, none of the above papers touched deployment strategy in RSN, which is practical and useful in RSN applications.

RSN is highly related to Wireless Sensor Networks (WSN) and many deployment strategies have been proposed in recent years in WSN. Yu et al. [8] and Luo et al. [9] applied grid to help deploy the sensor nodes according to detection features of the sensors. Xu et al. [10] and Mageid and Ramadan [11] investigated overall field coverage problems to deploy WSNs. Nevertheless all the above deployment strategies or placement algorithms are not considering the information integration, which is necessary in an RSN. Lin et al. [12] researched on the decision fusion rules for a WSN, but did not consider the deployment strategy of sensors. Aitsaadi et al. [13] derived

\section{Springer}

(c) 2013 Yang et al.; licensee Springer. This is an Open Access article distributed under the terms of the Creative Commons Attribution License (http://creativecommons.org/licenses/by/2.0), which permits unrestricted use, distribution, and reproduction in any medium, provided the original work is properly cited. 
a new WSN deployment strategy named multi-objective deployment algorithm based on evolutionary and neighborhood search algorithms. Kapnadak and Coyle [14] and Gogu et al. [15] determined the optimal spatial node densities for target detection. Xu et al. [16] and Ababnah and Natarajan [17] modeled the deployment based on the quantitative analysis of connectivity and network lifetime based on the collaborative detection model, respectively. However, most of these investigations have same assumption that the information sent from the sensors is perfectly recovered at the fusion center. This is not realistic for RSN as the transmitted information suffers channel distortions such as path-loss, log-normal, and multi-path fading. Thus, these deployment strategies are not suitable for RSN and new schemes are necessary.

In this study, we propose two graphical deployment strategies, namely, Hexagonal Deployment Strategy (HDS) and Diamond Deployment Strategy (DDS), to realize target detection with satisfying probability of false alarm and probability of target detection in RSN. In RSN, RSs send signals out and get echoes back for targets detection. When detecting targets and transmitting information, the RSs receive signals based on random channel. The target detection probability of each RS is obtained according to the position of the RS, independently. Based on the pass-loss fading channel environment, two fusion rules with Binary Transmission (BT) and without Binary Transmission (NBT) are derived to evaluate the performance of the two graphical deployment strategies in a multi-hop RSN. For better evaluating the two deployment strategies, the RS number and energy consumption for expected detection performance are researched. These two deployment strategies are applied to resourceconstrained RSN for reducing resource consumption and improving RSN performance in target detection at the same time. They are suitable for monitoring area of rectangular or area having the approximate shape of a rectangular.

The remainder of the article is as follows. Section 2 introduces the principles of deployment we use in this RSN and proposes the fading channel model and detection rules. Section 3 depicts deployment methods and processes with a finite number of RSs. Section 4 elaborates two decision fusion rules. Based on the simulation result, Section 5 analyzes performance evaluation of deployment methods. Finally, Section 6 draws the conclusion.

\section{Design principles and channel model}

Assume that there is a rectangular area $S(L \times W)$ under surveillance, where $L$ and $W$ are the length and width, respectively. $N$ RSs will be deployed to detect targets within this monitoring area. $N-1$ RSs individually radiates signals, receives echoes, and makes local decision whether there are targets in the monitoring area or not.
These $N-1$ RSs also transmit their independent decision to the fusion center (the remaining one node) through a number of relay nodes (selected from these $N-1$ sensors). The fusion center is placed to collect data and make a final decision. Our purpose is to investigate how to deploy these RSs with proper fusion rules so that satisfying detection performance and energy efficiency can be achieved.

In RSN, radio-frequency (RF) signals have unique attenuation characteristic, pass-loss model. Assume that the transmission power is $P_{t}$ and the receiving power is $P_{r}$, the model on the ground is given by

$$
P_{r} \propto \frac{P_{t}}{l^{\alpha}}
$$

where $l$ is the distance between transmission node and receiving node and $\alpha$ is the RF attenuation exponent. Due to multipath and other interference effects, $\alpha$ is typically in the range of 2 to 5 [18].

Each RS transmits a known waveform and receives the echoes from targets, independently. According to the pass-loss model, the power of received echo signal is given by

$$
P_{r} \propto \frac{P_{t} G \delta}{d^{2 \alpha}}
$$

where $d$ is the distance between RS and target, $G$ is the gain of radar antenna, and $\delta$ is radar cross section.

There are two hypotheses under test for each RS that either having a target $\left(H_{1}\right)$ or having no target $\left(H_{0}\right)$. Due to the above radar detection model, the two hypotheses $H_{0}$ and $H_{1}$ under test can be given by

$$
\begin{aligned}
& H_{1}: x_{k}=A_{r k}+n_{k} \\
& H_{0}: x_{k}=n_{k}
\end{aligned}
$$

where $x_{k}$ is the echo signal amplitude received by the $k$ th sensor, $k=1,2, \ldots, N-1, n_{k}$ is additive Gaussian noise with zero mean and variance $\sigma^{2}$ and $A_{r k}=\frac{\sqrt{G \delta} A_{t}}{d_{k}^{\alpha}}$ is the signal amplitude echoing to the $k$ th sensor, $A_{t}$ is transmitted signal amplitude, $d_{k}$ is the distance between target and the $k$ th sensor.

Assume that the targets emerge in the rectangular area homogeneously that the targets obey uniform distribution of the rectangular. According to the relationship between $d$ and $A_{r k}$, we know our $A_{r k}$ is a random variable unlike traditional test. For a fixed position RS, the probability density function of $A_{r k}$ can be written as $f\left(A_{r k}\right)$ which is related to the position of the RS. Assume that the $k$ th RS makes a binary decision $u_{k} \in\{+1,-1\}, P_{d k}=$ $P\left[u_{k}^{0}=+1 \mid H_{1}\right]$ represent probability of detection, $P_{f k}=$ $P\left[u_{k}^{0}=+1 \mid H_{0}\right]$ represent probability of false alarm. When there is just one target in our monitoring area, false alarm probability $P_{f k}$ and detection probability $P_{d k}$ can be derived by 


$$
\begin{aligned}
P_{f k}= & P\left\{x_{k}>T \mid H_{0}\right\} \\
= & \int_{T}^{\infty} \frac{1}{\sqrt{2 \pi} \sigma} \exp \left(-\frac{x_{k}^{2}}{2 \sigma^{2}}\right) d_{x_{k}} \\
P_{d k}= & P\left\{x_{k}>T \mid H_{1}\right\} \\
= & \int_{T}^{\infty} \int_{T_{1 k}}^{T_{2 k}} f\left(A_{r k}\right) \frac{1}{\sqrt{2 \pi} \sigma} \\
& \times \exp \left(-\frac{\left(x_{k}-A_{r k}\right)^{2}}{2 \sigma^{2}}\right) d_{A_{r k}} d_{x_{k}},
\end{aligned}
$$

where $T$ can be get according to the relationship between $P_{f k}$ and $T$ when the given $P_{f k}, T_{1 k}$, and $T_{2 k}$ are the minimum and maximum signal amplitude echoing to the $k$ th sensor. Obviously, the $P_{d k}$ is related to the position of the RS. When there are $M$ targets in the monitoring rectangular area, considering the influence among targets, we define the $k$ th local false alarm and detection probabilities as $P_{f k_{M}}=1-\left(1-P_{f k}\right)^{\frac{M}{4}+\frac{3}{4}}$ and $P_{d k_{M}}=\left(P_{d k}\right)^{\frac{M}{4}+\frac{3}{4}}$.

\section{Graphical deployment strategies}

In practice, we can usually regard the monitoring area as a rectangle for target detection. In this section, the two deployment strategies for detecting a rectangular region are proposed as follows.

\subsection{HDS}

The HDS is a strategy that place finite RSs to form mutually mosaic hexagons in the monitoring plane area. The following processes are taken.

1. Take the vertex $K$ of bottom left margin of this rectangle as a starting point and $R$ as the length of each segment, dividing equally the rectangle hemline. Starting with these equal division points, make rays with $60^{\circ}$ and $120^{\circ}$ until they intersect with boundary of this rectangle.

2. Take $K$ as a starting node and $\frac{\sqrt{3}}{2} R$ as the length of each segment, dividing equally the rectangle left boundary. Starting with these equal division nodes, make rays with $60^{\circ}$ and rays paralleling to the hemline of this rectangle until they intersect with boundary of this rectangle.

3. Now we need to select the proper points to place RSs there to form hexagons. First, pick out the points in the rectangle from these crossing points obtained according to above process. Second, in each odd row, respectively, taking the first point as the starting point, pick out every third point and then taking the third point as the starting point, pick out every third point. Third, in each even row, respectively, taking the first point as the starting point, pick out every third point and then taking the second point as the starting point, pick out every third point.
4. Finally, select the node which is closest to the geometric center of this rectangle to place a fusion center of RSN from nodes composing these hexagons and nodes in the center of these hexagons.

The number of sensors in the RSNs monitoring rectangular area for HDS is

$$
N_{1} \approx \frac{2}{3} \times\left[\frac{L}{R}\right] \times\left[\frac{W}{\frac{\sqrt{3}}{2} R}\right]
$$

where $N_{1}$ is a approximate number of sensors rather than a accurate one, $[*]$ is an integer of not less than $*$.

After the above placing process, a whole nodes' placement diagram is completed. Here, we use a $400 \times 300 \mathrm{~m}^{2}$ area (notice that the square is a special case of rectangle) as an example to exhibit the HDS. Figure 1 shows the graphical deployment of $N_{1}=30$ nodes in a $400 \times 300 \mathrm{~m}^{2}$ area using HDS.

\subsection{DDS}

The DDS is a strategy that place finite RSs to form mutually mosaic diamonds in the monitoring plane area. The following processes are used to deploy sensors in DDS.

1. Take the vertex $K$ of bottom left margin of this rectangle as a starting point and $R$ as the length of each segment, dividing equally the rectangle bottom boundary. Starting with these equal division points, make rays with $45^{\circ}$ and $135^{\circ}$ until they intersect with boundary of this rectangle.

2. Take $K$ as a starting point and $\frac{\sqrt{3}}{2} R$ as the length of each segment, dividing equally the rectangle left boundary. Starting with these equal division points, make rays with $45^{\circ}$ and rays paralleling to the

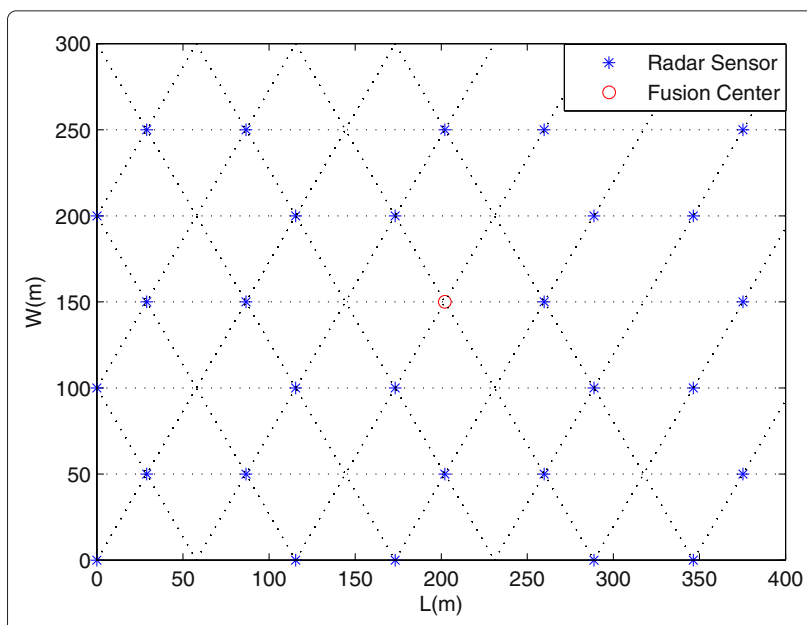

Figure 1 Array $N=30$ sensors in the area $400 \times 300 \mathrm{~m}^{2}$ using the HDS strategy. 
hemline of this rectangle until they intersect with boundary of this rectangle.

3. Select the nodes in the rectangle from these crossing nodes to place RSs there to form diamonds.

4. Finally, select the node which is closest to the geometric center of this rectangle to place a fusion center of RSN from nodes composing these diamonds and nodes in the center of these diamonds.

The number of sensors in the RSNs monitoring rectangular area for DDS is

$$
N_{2} \approx\left[\frac{L}{R}\right] \times\left[\frac{W}{\frac{R}{2}}\right],
$$

where the $N_{2}$ is not a accurate one, but a approximate number of sensors instead.

After the above placing process, a whole array nodes diagram is completed. Here, we use a $400 \times 300 \mathrm{~m}^{2}$ area as example to exhibit the DDS strategy. Figure 2 shows the graphical deployment of $N_{2}=30$ nodes in a $300 \times 400 \mathrm{~m}^{2}$ area using DDS.

\section{Decision fusion rules and energy analysis in multi-hop RSN}

$N-1$ RSs transmit a known signal and collect data generated according to the echo. They make local decisions (their independent decisions) according to these data and then transmit these decisions to a fusion center through several relay nodes over fading and noisy channels. The relay nodes are selected following a certain routing protocol. Each relay node sends the decision from its source node to the next node until it reaches the fusion center. The fusion center tries to decide whether or not to have a target in the monitoring area based on the received information. For the significantly improving of detection

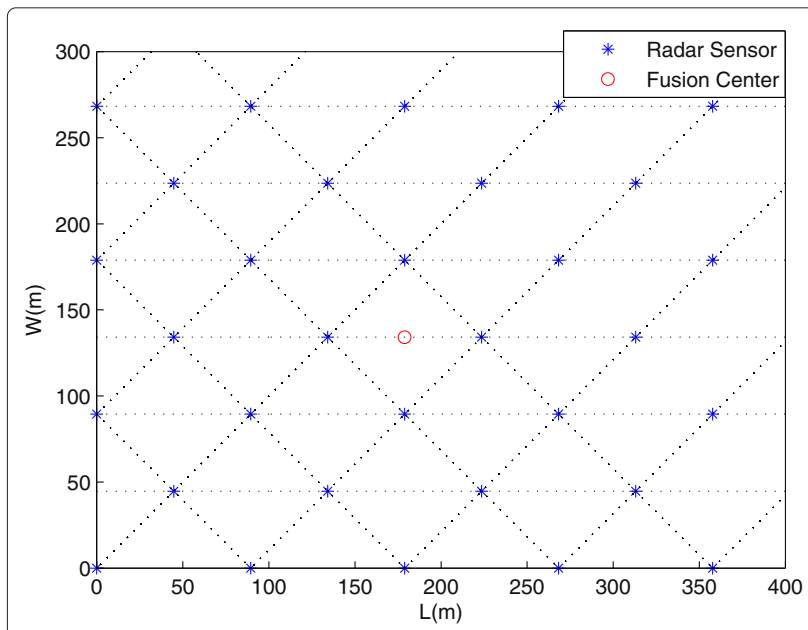

Figure 2 Array $N=30$ sensors in the area $400 \times 300 \mathrm{~m}^{2}$ using DDS strategy. performance, the fusion center uses likelihood ratio to fuse each sensor's local decision. In this section, we will describe two fusion rules based on different relay rules.

\subsection{Decision fusion rules with BT}

In this decision fusion rule, each relay node tries to retrieve the decision sent from its source node in spite of fading and noise distortion. These relay nodes make a binary decision when receiving signals. Assume that all the channels are independent of each other and each of them can be modeled as a path-loss channel. Noise in all channels are Gaussian with zero mean and variance $\sigma^{2}$ and are independent of each other.

The signal amplitude that every RS sends for detection is $A_{a}$ and for relay is $A_{b}$. Assume that $M_{k}$ denotes the number of relay nodes between the $k$ th local RS and the fusion center, with $k=1,2, \ldots, N-1$. The $h_{k}^{i}$ is the corresponding channel gain and $i=0,1, \ldots, M_{k}$ is the hop index. The process of target detection in $\mathrm{BT}$ is described below.

1. Every RS sends signal with amplitude $A_{a}$ out for detection and receives echo from target, independently.

2. According to the echoes, each RS individually makes a binary decision (local decision) $: u_{k}^{0}=+1$ is made if $H_{1}$ is decided, and $u_{k}^{0}=-1$ is made otherwise. They each sends signal $v_{k}^{0}=A_{b} u_{k}^{0}$ out for relay.

3. Local decisions made at $N-1 \mathrm{RSs}$ are transmitted over pass-loss fading channels to the fusion center through several relay nodes. Every relay node makes a binary decision which $u_{k}^{i}$ is either +1 or -1 and sends signal $v_{k}^{i}$ out. There

$$
\begin{aligned}
u_{k}^{i} & =\operatorname{sign}\left(v_{k}^{i-1} h_{k}^{i-1}+n_{k}^{i-1}\right) \\
v_{k}^{i} & =A_{b} u_{k}^{i}
\end{aligned}
$$

4. The decisions are sent to the fusion center, finally. Let $y_{k}$ denotes the input signal of the fusion center from the $k$ th RS, thus,

$$
y_{k}=A_{b} u_{k}^{M_{k}} h_{k}^{M_{k}}+n_{k}^{M_{k}}
$$

So, when $u_{k}^{M_{k}}$ is determined, $y_{k}$ obey the Gaussian distribution with mean $A_{b} u_{k}^{M_{k}} h_{k}^{M_{k}}$ and variance $\sigma^{2}$. Based on the received data $y_{k}$ for all RSs, the fusion center decides whether having a target or not.

Define $P_{d k}^{(c)}$ and $P_{f k}^{(c)}$ as the probability of detection and probability of false alarm, respectively, at the last relay.

$$
\begin{aligned}
& P_{d k}^{(c)}=P\left(u_{k}^{M_{k}}=+1 \mid H_{1}\right) \\
& P_{f k}^{(c)}=P\left(u_{k}^{M_{k}}=+1 \mid H_{0}\right)
\end{aligned}
$$

They are different from the local performance indices $P_{d k}$ and $P_{f k}$. 
The optimal LR-based fusion statistics for the multi-hop systems with BT is denoted by $\Lambda_{1}$. Given $P_{d k}^{(c)}$ and $P_{f k}^{(c)}$, the LR with the fusion statistic can be written as

$$
\begin{aligned}
\Lambda_{1}= & \frac{f\left(Y \mid H_{1}\right)}{f\left(Y \mid H_{0}\right)} \\
= & \prod_{k=1}^{N-1} \frac{f\left(y_{k} \mid H_{1}\right)}{f\left(y_{k} \mid H_{0}\right)} \\
= & \prod_{k=1}^{N-1} \frac{f\left(y_{k} \mid u_{k}^{M_{k}}=+1, H_{1}\right) P\left[u_{k}^{M_{k}}=+1 \mid H_{1}\right]+f\left(y_{k} \mid u_{k}^{M_{k}}=-1, H_{1}\right) P\left[u_{k}^{M_{k}}=-1 \mid H_{1}\right]}{f\left(y_{k}^{M_{k}}=+1, H_{0}\right) P\left[u_{k}^{M_{k}}=+1 \mid H_{0}\right]+f\left(y_{k} \mid u_{k}^{M_{k}}=-1, H_{0}\right) P\left[u_{k}^{M_{k}}=-1 \mid H_{0}\right]} \\
= & \prod_{k=1}^{N-1} \frac{P_{d k}^{(c)} e^{-\left(\left(y_{k}-h_{k}^{M_{k}} A_{b}\right)^{2} / 2 \sigma^{2}\right)}+\left(1-P_{d k}^{(c)}\right) e^{-\left(\left(y_{k}+h_{k}^{M_{k}} A_{b}\right)^{2} / 2 \sigma^{2}\right)}}{P_{f k}^{(c)} e^{-\left(\left(y_{k}-h_{k}^{M_{k}} A_{b}\right)^{2} / 2 \sigma^{2}\right)}+\left(1-P_{f k}^{(c)}\right) e^{-\left(\left(y_{k}+h_{k}^{M_{k}} A_{b}\right)^{2} / 2 \sigma^{2}\right)}} \\
= & \prod_{k=1}^{N-1} \frac{P_{d k}^{(c)}+\left(1-P_{d k}^{(c)}\right) e^{-\left(2 y_{k} h_{k}^{M_{k}} A_{b} / \sigma^{2}\right)}}{P_{f k}^{(c)}+\left(1-P_{f k}^{(c)}\right) e^{-\left(2 y_{k} h_{k}^{M_{k}} A_{b} / \sigma^{2}\right)}}
\end{aligned}
$$

\subsection{Decision fusion rules NBT}

In this fusion rule, we assume that relay nodes do not make binary decision when transmitting data. They simply forward the information from source nodes to the fusion center. Other conditions are the same as BT case. The process of target detection in NBT is described as follows.

1. Every RS sends signal with amplitude $A_{a}$ out for detection and receives echo from target, independently.

2. According to the echoes, each RS individually makes a binary decision (local decision) $: u_{k}^{0}=+1$ is made if $H_{1}$ is decided, and $u_{k}^{0}=-1$ is made otherwise. They each sends signal $v_{2 k}^{0}=A_{b} u_{k}^{0}$ for relay.

3. Local decisions made at $N-1$ RSs are transmitted over pass-loss fading channels to the fusion center through several relay nodes. Every relay node simply forward the information $v_{2 k}^{i}$ from source sensor.

$$
v_{2 k}^{i}=v_{2 k}^{i-1} h_{k}^{i-1}+n_{k}^{i-1}
$$

4. The decisions are sent to the fusion center, finally. The input signal of the fusion center from the $k$ th RS is

$$
\begin{aligned}
y_{2 k}= & h_{k}^{M_{k}} v_{2 k}^{M_{k}}+n_{k}^{M_{k}} \\
= & h_{k}^{M_{k}}\left[\ldots h_{k}^{2}\left(h_{k}^{1}\left(h_{k}^{0} v_{2 k}^{0}+n_{k}^{0}\right)+n_{k}^{1}\right)+n_{k}^{2} \ldots\right]+n_{k}^{M_{k}} \\
= & h_{k}^{M_{k}} \ldots h_{k}^{2} h_{k}^{1} h_{k}^{0} v_{2 k}^{0}+h_{k}^{M_{k}} \ldots h_{k}^{2} h_{k}^{1} n_{k}^{0}+h_{k}^{M_{k}} \ldots h_{k}^{2} n_{k}^{1} \\
& +h_{k}^{M_{k}} \ldots h_{k}^{3} n_{k}^{2}+\cdots+h_{k}^{M_{k}} n_{k}^{M_{k}-1}+n_{k}^{M_{k}}
\end{aligned}
$$

On account of that $n_{k}^{i}$ is additive Gaussian noise, when the $u_{k}^{0}$ is fixed, $y_{2 k}$ obeys the Gaussian distribution. We set $\mu_{k}=h_{k}^{M_{k}} \ldots h_{k}^{2} h_{k}^{1} h_{k}^{0} A_{b}$ and $\sigma_{k}^{2}=\left[\left(h_{k}^{M_{k}} \ldots h_{k}^{2} h_{k}^{1}\right)^{2}+\left(h_{k}^{M_{k}} \ldots h_{k}^{2}\right)^{2}+\cdots+\left(h_{k}^{M_{k}}\right)^{2}+(1)^{2}\right] \sigma^{2}$. When $u_{k}^{0}=1$ at the $k$ th local sensor, $y_{2 k}$ obeys the Gaussian distribution with mean $\mu_{k}$ and variance $\sigma_{k}^{2}$ and when $u_{k}^{0}=0$ at the $k$ th local sensor, $y_{2 k}$ obeys the Gaussian distribution with mean $-\mu_{k}$ and variance $\sigma_{k}^{2}$.

Due to $15, f\left(y_{2 k} \mid H_{1}\right)$ and $f\left(y_{2 k} \mid H_{0}\right)$ are decomposed into

$$
\begin{aligned}
f\left(y_{2 k} \mid H_{1}\right) & =f\left(y_{2 k} \mid u_{k}^{0}=+1, H_{1}\right) P\left(u_{k}^{0}=+1 \mid H_{1}\right)+f\left(y_{2 k} \mid u_{k}^{0}=-1, H_{1}\right) P\left(u_{k}^{0}=-1 \mid H_{1}\right) \\
& =P_{d k} f\left(y_{2 k} \mid u_{k}^{0}=1, H_{1}\right)+\left(1-P_{d k}\right) f\left(y_{2 k} \mid u_{k}^{0}=-1, H_{1}\right) \\
f\left(y_{2 k} \mid H_{0}\right) & =f\left(y_{2 k} \mid u_{k}^{0}=+1, H_{0}\right) P\left(u_{k}^{0}=+1 \mid H_{0}\right)+f\left(y_{2 k} \mid u_{k}^{0}=-1, H_{0}\right) P\left(u_{k}^{0}=-1 \mid H_{0}\right) \\
& =P_{f k} f\left(y_{2 k} \mid u_{k}^{0}=1, H_{0}\right)+\left(1-P_{f k}\right) f\left(y_{2 k} \mid u_{k}^{0}=-1, H_{0}\right)
\end{aligned}
$$


Therefore, in NBT situation, the LR can be written as

$$
\begin{aligned}
\Lambda_{2} & =\prod_{k=1}^{N-1} \frac{f\left(y_{k} \mid u_{k}^{0}=+1, H_{1}\right) P\left[u_{k}^{0}=+1 \mid H_{1}\right]+f\left(y_{k} \mid u_{k}^{0}=-1, H_{1}\right) P\left[u_{k}^{0}=-1 \mid H_{1}\right]}{f\left(y_{k} \mid u_{k}^{0}=+1, H_{0}\right) P\left[u_{k}^{0}=+1 \mid H_{0}\right]+f\left(y_{k} \mid u_{k}^{0}=-1, H_{0}\right) P\left[u_{k}^{0}=-1 \mid H_{0}\right]} \\
& =\prod_{k=1}^{N-1} \frac{P_{d k} e^{-\left(\left(y_{2 k}-\mu_{k}\right)^{2} / 2 \sigma_{k}^{2}\right)}+\left(1-P_{d k}\right) e^{-\left(\left(y_{2 k}+\mu_{k}\right)^{2} / 2 \sigma_{k}^{2}\right)}}{P_{f k} e^{-\left(\left(y_{2 k}-\mu_{k}\right)^{2} / 2 \sigma_{k}^{2}\right)}+\left(1-P_{f k}\right) e^{-\left(\left(y_{2 k}+\mu_{k}\right)^{2} / 2 \sigma_{k}^{2}\right)}} \\
& =\prod_{k=1}^{N-1} \frac{P_{d k}+\left(1-P_{d k}\right) e^{-\left(2 y_{2 k} \mu_{k} / \sigma_{k}^{2}\right)}}{P_{f k}+\left(1-P_{f k}\right) e^{-\left(2 y_{2 k} \mu_{k} / \sigma_{k}^{2}\right)}}
\end{aligned}
$$

\subsection{Energy analysis}

The total transmitting power $P$ can be divided into two main components: the power consumption of detecting target $P_{\text {det }}$ and the power consumption of forwarding decisions $P_{\text {for }}$. There $P=P_{\text {det }}+P_{\text {for }}$. The $P_{\text {det }}$ is the sum of power sent at each RS for target detection. The $P_{\text {for }}$ is the summation of power consumed at every relay node for forwarding the information from the $N-1$ RSs.

Therefore, the total energy consumption per bit for a fixed-rate system can be obtained as

$$
E_{b t}=\frac{P_{\mathrm{det}}+P_{\mathrm{for}}}{R_{\mathrm{b}}},
$$

where $R_{\mathrm{b}}$ is the bit rate.

In view of these $N-1 \mathrm{RSs}$ in our area of interest, the average energy consumption of every RS for transmitting is derived by

$$
E_{\mathrm{ave}}=\frac{E_{b t}}{N-1} .
$$

\section{Simulation results}

In this section, we analyze the performances of HDS, DDS, and RDS deployment in terms of probability of detection and energy efficiency with BT and NBT decision fusion rules. We place finite $\mathrm{RSs}$ in a $400 \times 300 \mathrm{~m}^{2}$ rectangular surveillance area as an example. The local detection probabilities $P_{d k}$ are generated mainly based on distances between sensors and target. As an illustration, set false alarm probability $P_{\mathrm{fa}}=0.001$ and local false alarm probability $P_{f k}=0.05$. The SNR is the average of SNR for detection and SNR for forwarding based on pass-loss fading channel.

Figure 3 compares the probability of detection versus channel SNR for three deployment strategies with different conditions. Besides HDS and DDS, we include random deployment strategy (RDS) for better performance comparison. Each point are obtained using $10^{5}$ Monte Carlo runs. The simulation result shows the following facts:

1. It can be seen that the performance for BT fusion rules is better than that for NBT fusion rules.
2. Both HDS and DDS are better than RDS in terms of detection probability, and DDS is superior to HDS, no matter in $\mathrm{BT}$ or NBT. It means that arraying nodes regularly can get higher detection probability than deploying them randomly. Both HDS and DDS are efficient in target detection.
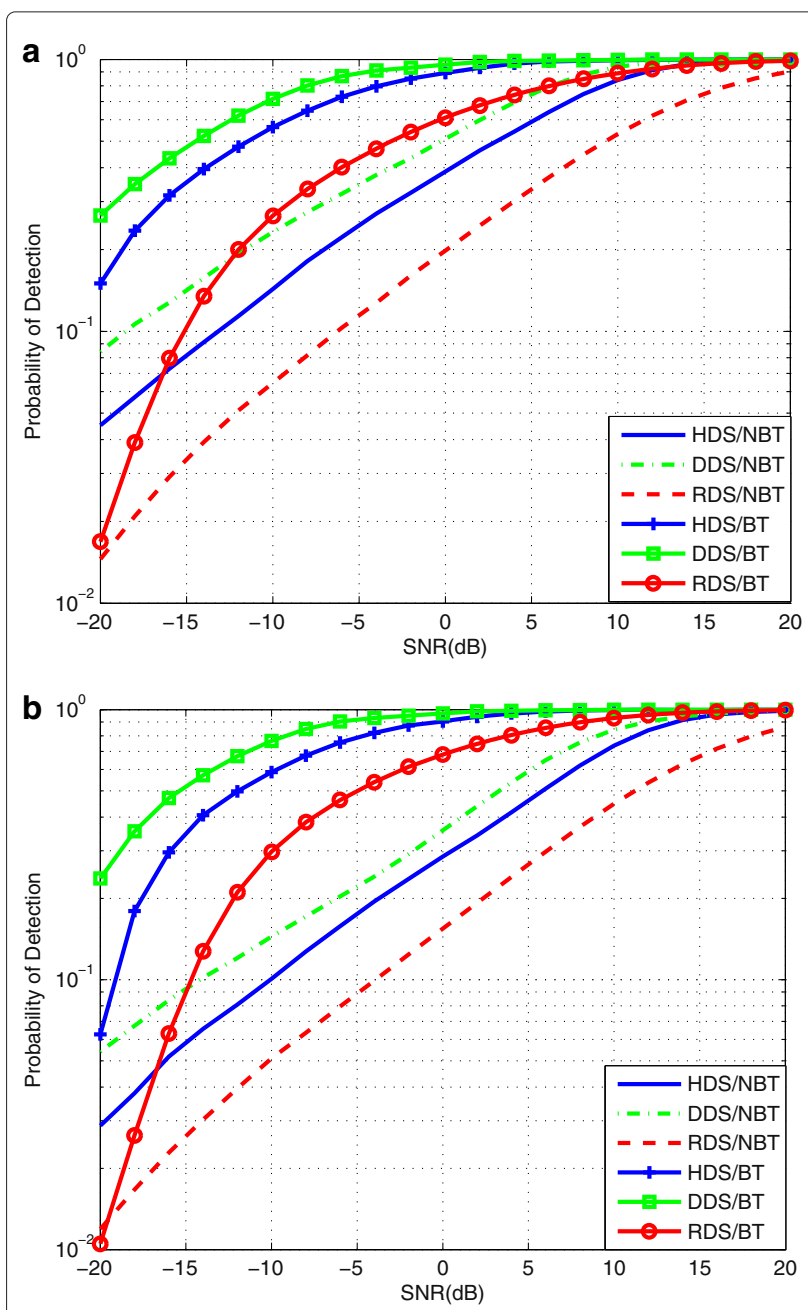

Figure 3 Probability of detection versus channel SNR using HDS/DDS/RDS and BT/NBT for pass-loss fading channels (a) $N=20$, (b) $N=30$. 

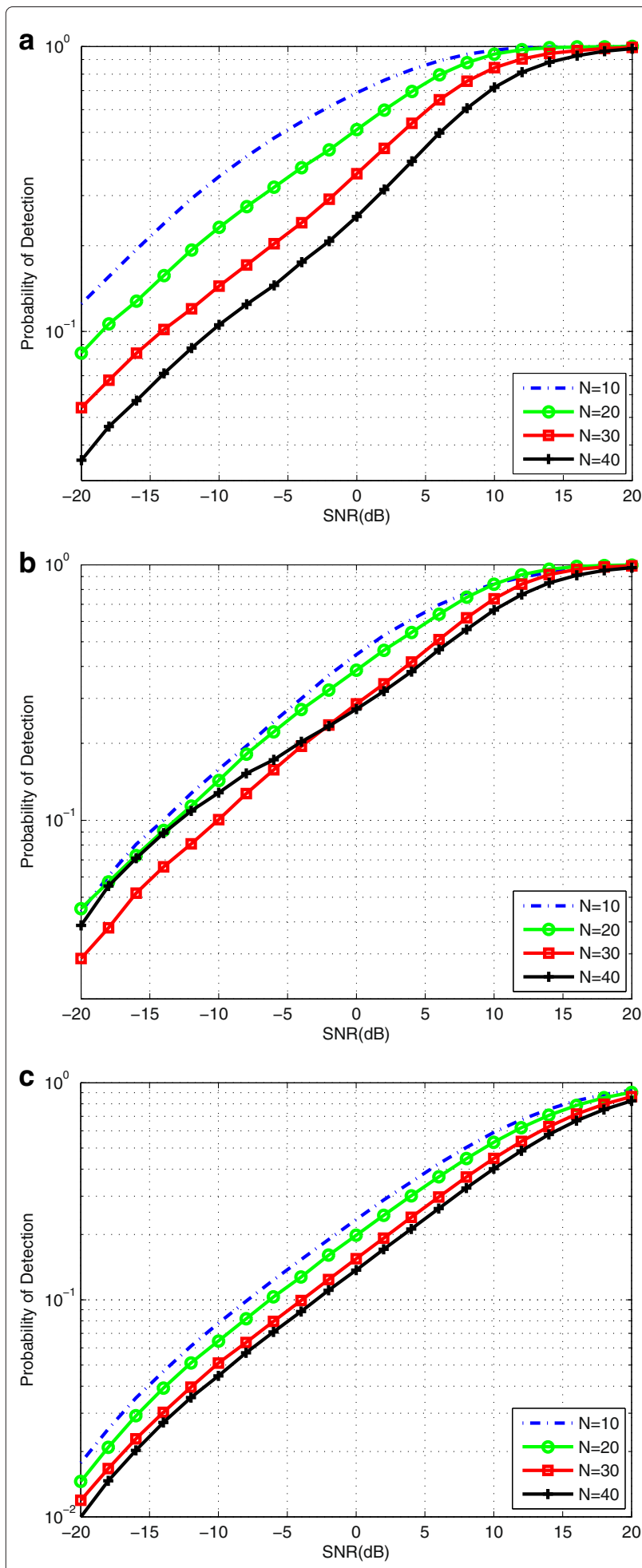

Figure 4 Probability of detection versus channel SNR in NBT for four sensor numbers, using (a) DDS, (b) HDS, (c) RDS.
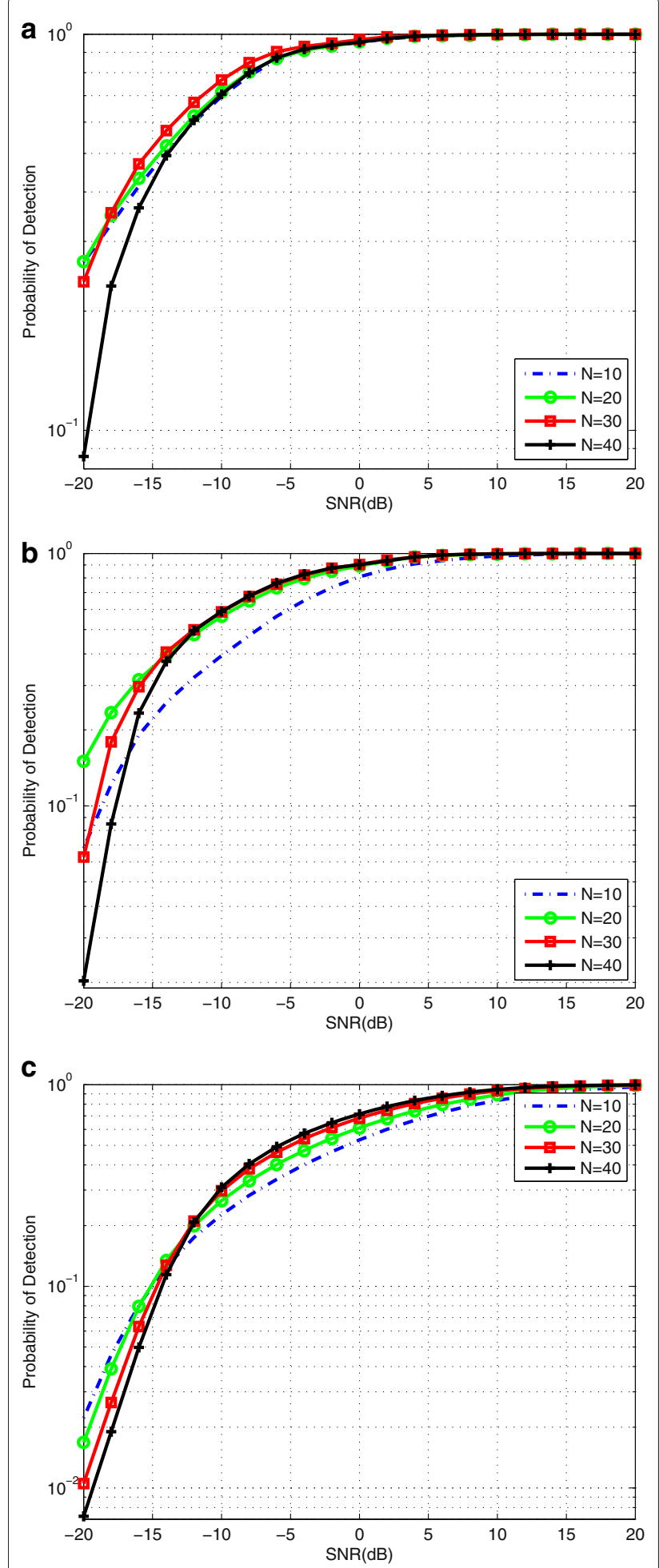

Figure 5 Probability of detection versus channel SNR in BT for four sensor numbers, using (a) DDS, (b) HDS, (c) RDS. 


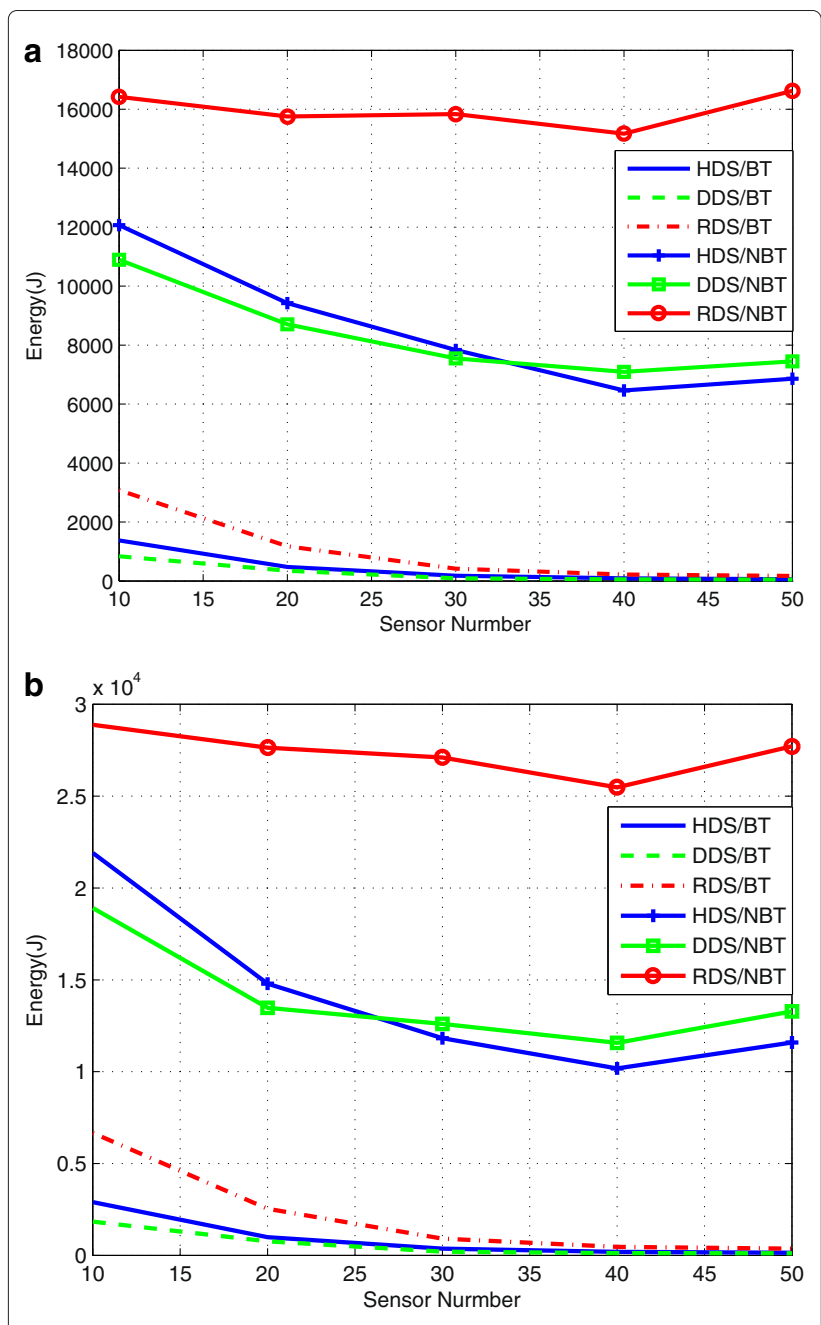

Figure 6 The total average transmitting energy of every sensor versus sensor number $N$ for three deployment strategies in two fusion rules with (a) $P_{d}=0.9$, (b) $P_{d}=0.95$. rules with system detection probability $P_{d}=0.9$, while Figure $6 \mathrm{~b}$ applies $P_{d}=0.95$. Figures $6 \mathrm{a}$,b shows that the BT fusion rules consume fewer energy than the NBT fusion rules. In BT, the energy consumption decreases as the increase of the RS number and DDS is more energy efficient than HDS. RDS consumes the most energy. While in NBT, when the RS number is smaller than 35 at $P_{d}=0.9$ and smaller than 25 at $P_{d}=0.95$, DDS is more energy efficient than HDS. However, as the RS number grows, HDS out performs DDS. RDS is still the wrest in energy efficiency. Thus, we may conclude that among DDS/HDS/RDS and BT/NBT, DDS with BT fusion rule is the best for target detection in RSN due to the high probability of detection, high energy efficiency, and the optimum RS.

\section{Conclusion}

In this article, we propose two deployment strategies, namely, HDS and DDS to deploy finite RSs to achieve a higher expected detection probability with low energy consumption to satisfy the target detection performance in RSN. Simulation results show that under same channel SNR, the DDS achieves highest probability of detection, the HDS gets a lower one and the RDS is lowest no matter in BT and NBT. To achieve the highest detection performance, there is an optimum choice of the sensor number. It is not the more the better. When the number of sensors is 30, RSN gets highest probability of detection in DDS and BT. The DDS consumes less energy than HDS in multi-hop RSN with pass-loss fading channel. The two deployment strategies both are more energy efficient than RDS. Due to the high detection probability, high energy efficiency and the optimum RS, DDS with BT fusion rule is the best among DDS/HDS/RDS and BT/NBT for target detection in RSN.

\section{Competing interests}

The authors declare that they have no competing interests.

\section{Acknowledgements}

This study was supported by the National Natural Science Foundation of China under Young Scientists Fund 61102140.

Received: 29 November 2012 Accepted: 1 February 2013 Published: 2 March 2013

\section{References}

1. J Liang, Q Liang, Design and analysis of distributed radar sensor networks. IEEE Trans. Parallel Distrib. Syst. 22(11), 1926-1933 (2011)

2. S Haykin, in 1st IEEE International Workshop on Computational Advances in Multi-Sensor Adaptive Processing. Cognitive radar networks (Nashville April, 2005), pp. 1-3

3. PK Dutta, AK Arora, SB Bibyk, in Fifth International Conference on Information Processing in Sensor Networks. Towards radar-enabled sensor networks (Nashville, TN April, 2006), pp. 467-474

4. HD Ly, Q Liang, in 2006 IEEE Military Communications Conference. Spatial-temporal-frequency diversity in radar sensor networks (Washington, DC, October, 2006), pp. 1-7 
5. HD Ly, Q Liang, in 2007 IEEE Military Communications Conference. Collaborative multi-target detection in radar sensor networks (Orlando, FL, October, 2007), pp. 1-7

6. H Deng, in 2010 IEEE 10th International Conference on Signal Processing (ICSP). Target detection with distributed radar sensor networking systems (DRASENS) (Beijing, October, 2010), pp. 1951-1954

7. H Shu, Q Liang, in 2007 IEEE Radio and Wireless Symposium. Data fusion in a multi-target radar sensor network (Long Beach, CA, January, 2007), pp. 129-132

8. S Yu, R Wang, H Xu, W Wan, Y Gao, Y Jin, in IET International Conference on Smart and Sustainable City. WSN nodes deployment based on artificial fish school algorithm for traffic monitoring system (Shanghai, July, 2011), pp. 1-5

9. H Luo, Z Liu, F Xue, in 2nd International Conference on Future Computer and Communication, vol. 1. A deployment strategy for target surveillance sensor networks based on acoustic energy measurements (Wuhan, May, 2010), pp. 686-690

10. $\mathrm{KXu}, \mathrm{Q}$ Wang, H Hassanein, $\mathrm{G}$ Takahara, in IEEE International Conference on Wireless And Mobile Computing, Networking And Communications, vol. 1. Optimal wireless sensor networks (WSNs) deployment: minimum cost with lifetime constraint, (August, 2005), pp. 22-24

11. SA Mageid, RA Ramadan, in International Conference on Autonomous and Intelligent Systems. Efficient deployment algorithms for mobile sensor networks (Povoa de Varzim, June, 2010), pp. 1-6

12. $Y$ Lin, $B$ Chen, $P K$ Varshney, Decision fusion rules in multi-hop wireless sensor networks. IEEE Trans. Aerospace Electron. Syst. 41(2), 475-488 (2005)

13. N Aitsaadi, N Achir, K Boussetta, G Pujolle, in 2010 IEEE International Conference on Communications (ICC). Multi-objective WSN deployment: quality of monitoring, connectivity and lifetime (Cape Town, May, 2010), pp. 1-6

14. V Kapnadak, EJ Coyle, in 8th Annual IEEE Communications Society Conference on Sensor, Mesh and Ad Hoc Communications and Networks (SECON). Optimal non-uniform deployment of sensors for detection in single-hop wireless sensor networks (Salt Lake City, UT, June, 2011), pp. 89-97

15. A Gogu, D Nace, Y Challal, in 14th International conference on Telecommunications Network Strategy and Planning Symposium (NETWORKS). A note on joint optimal transmission range assignment and sensor deployment for wireless sensor networks (Warsaw, September 2010), pp. 1-6

16. KXu, H Hassanein, G Takahara, Q Wang, Relay node deployment strategies in heterogeneous wireless sensor networks. IEEE Trans. Mob. Comput. 9(2), 145-159 (2011)

17. A Ababnah, B Natarajan, in 2009 IEEE Global Telecommunications Conference. Optimal sensor deployment for value-fusion based detection (Honolulu, HI, November-December, 2009), pp. 1-6

18. F Zhao, L Guibas. Wireless Sensor Networks: An Information Processing Approach (Morgan Kaufmann New York, 2004), p. 7

\section{doi:10.1186/1687-1499-2013-55}

Cite this article as: Yang et al: Graphical deployment strategies in radar sensor networks (RSN) for target detection. EURASIP Journal on Wireless Communications and Networking 2013 2013:55.

\section{Submit your manuscript to a SpringerOpen ${ }^{\circ}$ journal and benefit from:}

- Convenient online submission

- Rigorous peer review

- Immediate publication on acceptance

- Open access: articles freely available online

- High visibility within the field

- Retaining the copyright to your article

Submit your next manuscript at $\boldsymbol{\nabla}$ springeropen.com 